\title{
Endobronchial hamartoma presenting as massive haemoptysis
}

\author{
R.A. Sharkey, E.M.T. Mulloy, S. O'Neill
}

Endobronchial hamartoma presenting as massive haemoptysis. R.A. Sharkey, E.M.T. Mulloy, S. O'Neill. COERS Journals 1996.

ABSTRACT: A 53 year old man presented with massive haemoptysis, and chest radiography revealed a left suprahilar mass. At thoracotomy he was found to have a nodular mass in the left upper lobe, which was attached to a peripheral bronchus. Histology confirmed a pulmonary hamartoma.

Eur Respir J., 1996, 9, 2179-2180.
Dept of Respiratory Medicine, Beaumont Hospital, Dublin, Ireland.

Correspondence: S. O'Neill, Dept of Respiratory Medicine, Beaumont Hospital, Dublin 9, Ireland

Keywords: Endobronchial, haemoptysis, hamartoma

Received: January 161995

Accepted after revision March 41996
Pulmonary hamartomas are rare benign tumours, of which only $3-20 \%$ are endobronchial in nature [1-4]. Massive haemoptysis is a very unusual mode of presentation of pulmonary hamartomas, with, to our knowledge, only one previously reported case [5]. We describe a patient with an endobronchial hamartoma, who presented with massive haemoptysis.

\section{Case report}

A 53 year old man was admitted to our hospital with a 5 day history of increasing frank haemoptysis. He had an episode of massive haemoptysis after admission, producing $600 \mathrm{~mL}$ of fresh blood. He had no associated dyspnoea or chest pain. He had a chronic cough productive of mucoid sputum, but an otherwise uneventful past history. He was a very fit soldier, with a 7 pack-year history of smoking until $10 \mathrm{yrs}$ previously. There was no familial history of respiratory disease.

Physical examination revealed a healthy looking man with a pulse rate of 90 beats $\mathrm{min}^{-1}$, blood pressure 110/70 $\mathrm{mmHg}$, respiratory rate 18 breaths $\cdot \mathrm{min}^{-1}$, and temperature $36^{\circ} \mathrm{C}$. Physical examination was otherwise normal. Investigations showed a normal full blood count and urea and electrolyte levels. Blood gas values whilst breathing room air were: arterial oxygen tension $\left(\mathrm{Pa}_{\mathrm{a}} \mathrm{O}_{2}\right) 8.9 \mathrm{kPa}$; arterial carbon dioxide tension $\left(\mathrm{Pa}_{\mathrm{a}} \mathrm{CO}_{2}\right) 5.6 \mathrm{kPa}$; $\mathrm{pH} 7.42$; and arterial oxygen saturation $\left(\mathrm{Sa}_{2} \mathrm{O}_{2}\right) 94 \%$. Chest radiography showed a left suprahilar mass, which appeared to be irregular and noncalcified. Electrocardiogram was normal apart from left axis deviation. The patient had a bronchoscopy performed under local anaesthetic, which demonstrated blood throughout the tracheobronchial tree. The blood appeared to be emanating from the left upper lobe bronchus. Repeat bronchoscopy was carried out when the haemoptysis had settled, and this revealed a pulsatile lesion in the anterior segment of the left upper lobe. No biopsies were performed due to the risk of haemorrhage. A computed tomography (CT) scan of the thorax showed a mass in the medial aspect of the left upper lobe close to the left pulmonary artery (fig. 1).

Left posterolateral thoracotomy was performed, which revealed a nodular mass in the left upper lobe that was attached to a peripheral bronchus, but not to the pulmonary artery. A left upper lobectomy was performed, with sampling of hilar nodes. Macroscopically, the mass was whitish-tan in appearance, well circumscribed and measuring $2 \times 2 \times 1.5 \mathrm{~cm}$, with evidence of recent haemorrhage in the surrounding lung parenchyma. Microscopically, the mass was composed of lobules of hyaline cartilage intermixed with compressed glandular epithelium, which was arising from the bronchus. A diagnosis of endobronchial hamartoma was made. No clear source of haemorrhage could be identified, due to extensive tissue destruction by intraparenchymal haemorrhage. The hilar lymph nodes were normal. Lobectomy rather than enucleation of the tumour was performed in this case, because of the extensive parenchymal haemorrhage and risk of further bleeding. The patient made an excellent postoperative recovery and remains well 1 year later.

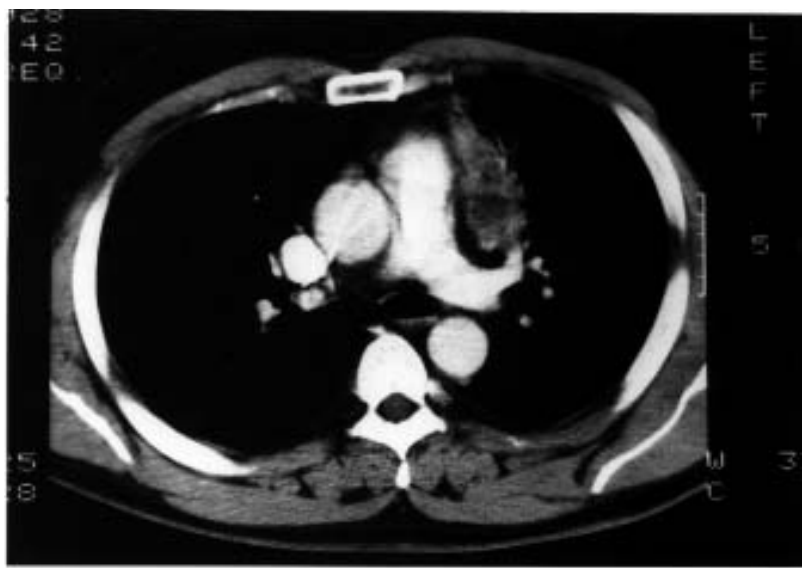

Fig. 1. - Contrast computed tomography (CT) scan of the thorax showing a mass in the medial aspect of the left upper lobe close to the left pulmonary artery. 


\section{Discussion}

Pulmonary hamartoma is an uncommon benign tumour [6]. It consists of an admixture of cartilage, loose collective tissue, fat, bone, epithelial clefts and gland-like epithelial aggregates. The median age of presentation is $50-60$ yrs of age, with a male:female ratio of $3-5: 1$. The majority of hamartomas are parenchymal and are often located in the periphery of the lung, unlike our patient who had a more central lesion. Because of their peripheral location, they tend to be asymptomatic and are usually found incidentally on chest radiographic examination. When the tumour becomes large enough, it may obstruct a bronchus causing atelectasis and recurrent pneumonia. Although hamartomas are almost always benign, malignant transformation and a tendency to invasion have been reported but are extremely rare [7].

Endobronchial hamartomas are unusual, accounting for approximately 3-20\% of all pulmonary hamartomas [1-4]. Initially, it was thought that endobronchial and intraparenchymal tumours were histogenetically different [8]. However, it is now accepted that both intraparenchymal and endobronchial lesions are similar tumours of primitive bronchial mesenchymal tissue, which has the capacity to differentiate toward multiple mature mesenchymal components [3]. The tumour in this case was visible on bronchoscopy, and both macroscopically and microscopically arose from a peripheral bronchus. The endobronchial tumours may be polypoid or pedunculated, and have a histological appearance similar to that of parenchymal hamartomas. The commonest presentation of such tumours are cough, dyspnoea and pyrexia. Haemoptysis can occur, but it is usually scanty. Presumably, in this case, the haemoptysis was due to the tumour eroding an adjacent blood vessel, since the tumour itself was relatively nonvascular.

Because the clinical and radiological features of endobronchial hamartomas are frequently indistinguishable from those of a carcinoma, histological examination is necessary for a definitive diagnosis. Peripheral hamartomas are frequently not visible on bronchoscopy. The introduction of fine needle aspiration biopsy offers a valuable alternative to diagnostic thoracotomy in peripheral hamartomas, with a diagnostic yield of up to $85 \%[4,9]$.
In the case of localized endobronchial hamartomas, removal via the bronchoscope has been reported without recurrence of the tumour. Where a firm diagnosis is made preoperatively, surgical removal may not be necessary unless symptoms are present, or tumour expansion is noted [4]. In this case, biopsy was not attempted because of the pulsatile nature of the tumour and its proximity to the pulmonary artery. The presence of life-threatening haemoptysis necessitated pulmonary resection without a firm preoperative diagnosis.

Acknowledgements: The authors acknowledge P. Broe, Beaumont Hospital, for performing the surgery, and D. Royston, Beaumont Hospital, for performing the histological examination.

\section{References}

1. Mateson EM. Relationship between intrapulmonary and endobronchial cartilage-containing tumors (so-called hamartoma). Thorax 1965; 20: 447-461.

2. Bergh NP, Hafstrom LO, Scherson T. Hamartoma of the lung: with special reference to the endobronchial localization. Scand J Respir Dis 1967; 48: 201-207.

3. Tomashefski JF. Benign endobronchial mesenchymal tumours: their relationship to parenchymal pulmonary hamartomas. Am J Surg Pathol 1982; 6: 531-540.

4. Hansen CP, Holtveg H, Francis D, Rasch L, Bertelsen S. Pulmonary hamartoma. J Thorac Cadiovasc Surg 1992; 104: 674-678.

5. Kleinman J, Zirkin H, Feuchtwanger MM, Hertzanu Y, Walfisch S. Benign hamartoma of the lung presenting as massive hemoptysis. J Surg Oncol 1986; 33: 38-40.

6. McDonald JR, Harrington SW, Clagett OT. Hamartoma (often called chondroma) of the lung. J Thorac Surg 1945; 14: 128-143.

7. Poulsen JT, Jacobsen M, Francis D. Probable malignant transformation of a pulmonary hamartoma. Thorax 1979; 34: 557-558.

8. Spencer H. Pathology of the Lung. Vol. 2 (3rd edn). New York, Pergamon Press, 1977; p. 892.

9. Hamper UM, Khouri NF, Stitik FP, Siegelman SS. Pulmonary hamartoma: diagnosis by transthoracic needleaspiration biopsy. Radiology 1985; 155: 15-18. 